\title{
Sex Differences of the Natriuretic Peptide Polymorphism Associated With Angiographic Coronary Atherosclerosis
}

\author{
Terry Y. Li ${ }^{a}$, M. Yat Tse ${ }^{a}$, Stephen C. Panga, d, Catherine S. McLellan ${ }^{b}$, Will D. King, \\ Amer M. Johria, b, d
}

\begin{abstract}
Background: Polymorphisms within natriuretic peptide (NP) genes have been associated with clinical outcomes for cardiovascular disease (CVD), but no previous study has compared the effect of these polymorphisms between men and women. This study aimed to investigate the association between single nucleotide polymorphisms (SNPs) in key genes of the NP system and coronary angiographic outcomes, with the focus on the sexual dimorphism in the effects of these SNPs.

Methods: Patients undergoing clinically indicated coronary angiography ( $\mathrm{n}=513,328$ men and 185 women) were consented and genotyped for NPPA rs5065, NPPB rs198389 and NPR2 rs10758325. Patients were stratified into having normal coronaries, non-obstructive coronary artery disease (CAD) or obstructive CAD, based on the highest stenosis in any epicardial artery. Average luminal narrowing across all four arteries was derived to represent the overall atherosclerotic burden.
\end{abstract}

Results: The frequency of $N P P B$ rs198389 AA genotype was significantly higher in women with obstructive $\mathrm{CAD}(\mathrm{P}=0.014)$. The same association was not observed in males. With respect to atherosclerotic burden, an association was found between the AA genotype and average luminal narrowing in women $(\mathrm{P}=0.005)$, but not in men.

Conclusions: The current study identified an association between an SNP of the $N P P B$ gene and coronary atherosclerotic burden through angiographic evidence in women but not in men. These results suggest that B-type natriuretic peptide (BNP) may have more important involvement in the development of CAD in women compared to men, and as such, genotyping of the $N P P B$ gene may serve as a potential

Manuscript accepted for publication February 06, 2017

aDepartment of Biomedical and Molecular Sciences, Queen's University, 18 Stuart Street, Kingston, ON, Canada

bDivision of Cardiology, Department of Medicine, Queen's University, 94 Stuart Street, Kingston, ON, Canada

'Department of Public Health Sciences, Queen's University, 62 Fifth Field Company Lane, Kingston, ON, Canada

${ }^{\mathrm{d} C}$ Corresponding Author: Amer M. Johri, Division of Cardiology, Department of Medicine, Queen's University, Etherington Hall Rooms 3032-3043, 94 Stuart Street, Kingston, ON, K7L 3N6, Canada. Email: amerschedule@gmail.ca; Stephen C. Pang, Department of Biomedical and Molecular Sciences, Queen's University, Botterell Hall Room 850, 18 Stuart St., Kingston, ON, K7L 3N6, Canada.Email: pangsc@queensu.ca

doi: https://doi.org/10.14740/cr523w biomarker to identify women with high risk for CAD.

Keywords: Coronary artery disease; Natriuretic peptide system; BNP; SNP; Sex difference

\section{Introduction}

Coronary artery disease (CAD) is the leading cause of morbidity and mortality worldwide. Although our understanding of the pathogenesis of atherosclerosis is not fully determined, $\mathrm{CAD}$ is well known to result from build-up of plaque in coronary arteries, leading to narrowing of these vessels.

Previous studies have demonstrated significant differences in diagnosis and development of CAD between men and women. Compared to men, women were found to have a lower prevalence of obstructive CAD, but those women who were diagnosed with obstructive $\mathrm{CAD}$ had higher rates of adverse outcomes than men [1-3]. In the presence of non-obstructive CAD, microvascular dysfunction, endothelial dysfunction and other processes that predict adverse outcome appear more frequent in women than in men [4]. It is unknown if these striking differences in CAD prevalence and outcomes are related to differences in the underlying genetic expression of hormonal circulatory systems involved in myocardial and vascular regulation.

The natriuretic peptide system plays an important role in hemodynamic regulation and its relation to heart disease has become of increasing importance lately with the advent of novel pharmaceuticals [5]. The natriuretic peptide system consists of three members, atrial natriuretic peptide (ANP), Btype natriuretic peptide (BNP) and C-type natriuretic peptide (CNP), encoded by $N P P A, N P P B$ and $N P P C$ genes, respectively. They exert their effects by binding to high-affinity receptors that are expressed on the surface of target cells. Natriuretic peptide receptors A and B mediate the majority of the cardiovascular effects of the natriuretic peptides. Natriuretic peptide receptor $\mathrm{C}$ is the clearance receptor, binding all three natriuretic peptides for internalisation and degradation. These receptors are encoded by NPR1, NPR 2 and NPR 3 genes, respectively.

Circulating levels of ANP and BNP are elevated in patients with ventricular hypertrophy and congestive heart failure [6], making them useful tools in assessing the degree of cardiac dysfunction and severity of left ventricular failure. Levels of BNP and N-terminal BNP (NT-proBNP) have consistently 
been found to be independent risk markers for both morbidity and mortality in post myocardial infarction and heart failure patients. On the other hand, newly approved heart failure medications work through the natriuretic peptide system to promote vasodilation and natriuresis, stimulating further interest in response to therapies that modify this system [5].

Numerous single nucleotide polymorphisms (SNPs) of NPPA and NPPB have previously been found to be associated with cardiovascular outcomes. Ellis et al (2011) reported that minor alleles of $N P P A$ rs5065, NPPB rs632793, rs198388, rs198389 and NPR2 rs10758325 are associated with a lower rate of hospital readmission related to all CVDs [7]. However, no previous study has examined the association between SNPs and angiographic CAD specifically, nor differences between males and females.

The current study aimed to investigate three SNPs within $N P P A, N P P B$ and NPR2 genes, namely rs5065, rs198389 and rs10758325, for their association with the severity of CAD through angiographic evidence. More importantly, we were interested in determining whether the effects of these SNPs on CAD differ between males and females.

\section{Materials and Methods}

\section{Patient cohort}

Written, informed consent was obtained from each participant recruited. The study protocol conforms to the ethical guidelines of the 1975 Declaration of Helsinki. The study was approved by the Health Sciences and Affiliated Teaching Hospitals Research Ethics Board at Queen's University (DMED-1365-11). The inclusion criteria for this study were comprised of patients undergoing an elective coronary angiogram at the Kingston General Hospital (KGH) Cardiovascular Lab (Kingston, Ontario). Patients were excluded only if there was a contra-indication for the blood sample collection. Patients were referred to undergo angiography due to one or more of the following indications: angina, positive electrocardiogram (ECG) test, positive (stress) imaging test, and previously known CAD.

\section{Angiography}

Coronary angiography was performed according to the standard Judkins method using a GE System 2000 (GE Healthcare) by one of four experienced interventional cardiologists. Percentage luminal diameter narrowing in each of the four major coronary arteries (left main, left anterior descending, circumflex and right coronary artery) was recorded. Patients were stratified into three categories: normal coronary (luminal narrowing $<20 \%$ in all epicardial arteries), non-obstructive CAD (luminal narrowing $\geq 20$ and $<70 \%$ in any epicardial artery or $\geq 20 \%$ and $<50 \%$ in the left main artery) and obstructive CAD (luminal narrowing $\geq 70 \%$ in any epicardial artery or $\geq 50 \%$ in the left main artery). Average luminal narrowing across all four vessels was also acquired to create a continuous variable that represents the overall coronary plaque burden.

\section{SNP selection}

SNPs of the natriuretic peptide system were selected using the following criteria: 1) have been identified in previous studies as associated with circulating natriuretic peptide level or cardiovascular outcomes and 2) have a minor allele frequency $>$ 10\%. The SNPs selected were NPPA rs5065, NPPB rs 198389 and NPR2 rs 10758325.

\section{DNA extraction and genotyping}

Whole blood was collected during the angiography procedure. White blood cells were isolated from the whole blood samples and digested using cell lysis buffer and proteinase $\mathrm{K}$ (5-PRIME, VWR Canada). Genomic DNA was precipitated using isopropanol, isolated and rehydrated with Tris buffer. DNA samples were diluted to $5 \mathrm{ng} / \mu \mathrm{L}$ prior to genotyping. Genotyping was performed using TaqMan SNP genotyping assays according to the manufacturer's protocol (Applied Biosystems, Foster City, California, USA) on a LightCycler ${ }^{\complement} 480$ II Real-Time PCR System (LC480-II; Roche Applied Science, Laval, Quebec, Canada).

\section{Statistical analysis}

Associations between natriuretic peptide SNP genotypes and CAD severity category were tested using a log-binomial regression model controlling for age and sex, where the relative risk (RR) was reported. Associations between natriuretic peptide SNP genotypes and average luminal narrowing were tested using a linear least squares regression model controlling for age and sex. The effect estimate $(\beta)$ was reported to represent the adjusted difference in overall plaque burden. The differences in SNP effects between sexes were tested with an interaction term in aforementioned models. The log-binomial regression model was also used to test for associations between natriuretic peptide SNPs and clinical characteristics including hypertension, type II diabetes and history of MI. A P-value of $\leq 0.05$ was deemed to be statistically significant. All analyses were performed using SAS Enterprise Guide 6.1 (SAS Institute Inc., Cary, NC, USA).

\section{Results}

\section{Power calculation}

A priori, a patient sample size of 500 was planned and prevalence of obstructive CAD was estimated to be $50 \%$ according to study definitions (luminal narrowing $\geq 70 \%$ in any epicardial artery or $\geq 50 \%$ in the left main artery). SNPs will be dichotomized with at risk genotype prevalence ranging from $25 \%$ to $65 \%$. This sample provides $80 \%$ power to detect RRs of 1.3 and greater for each SNP (two-tailed significance of 0.05) [8]. A higher proportion of males than females were expected in 
Table 1. Participant Characteristics $(n=513)$

\begin{tabular}{ll}
\hline Characteristics & Mean \pm SD or percent \\
\hline Age (years) & $65.3 \pm 11.1$ \\
Sex male & $63.94 \%$ \\
Caucasian & $98.2 \%$ \\
BMI $\left(\mathrm{kg} / \mathrm{m}^{2}\right)$ & $30.3 \pm 6.3$ \\
Current smoker & $18.8 \%$ \\
Hypertension & $65.1 \%$ \\
Diabetes & $31.3 \%$ \\
Previous MI & $28.0 \%$ \\
Family history of heart diseases & $67.7 \%$ \\
CAD categories & \\
\multicolumn{1}{c}{ Normal coronary } & $22.6 \%$ \\
\multicolumn{1}{l}{ Non-obstructive CAD } & $21.3 \%$ \\
\hline
\end{tabular}

the sample and detectable RRs are 1.4 in males $(n=320)$ and 1.5 in females $(n=180)$.

\section{Participant characteristics}

Characteristics of the study cohort $(n=513)$ are described in Table 1 . The mean age of participants was 65.3 years, and $64 \%$ of them were male. The majority of participants were Caucasians, with four Asian descendants, two African descendants, two Canadian First Nation individuals and one mixed race. Just over half of the participants $(56.1 \%)$ were diagnosed with obstructive CAD, while $22.6 \%$ were diagnosed with no or minimal disease (normal coronary arteries).

The distribution of average luminal diameter narrowing is presented in Figure 1. It is heavily skewed to the left because of the large number (124) of patients with $0 \%$ narrowing in all four vessels. Thus, only patients diagnosed with CAD (luminal narrowing $\geq 20 \%$ in any epicardial artery) $(n=387)$ were included in the linear least squares model to assess overall coronary plaque burden. The mean of average luminal narrowing among these patients was $39.5 \%( \pm 22.3)$.

\section{Genetic statistics}

Genotype frequencies for each natriuretic peptide system SNP are shown in Table 2. In our study population, all three SNPs were in Hardy-Weinberg equilibrium (P-values were 0.09, 0.23 and 0.78 for rs5065, rs198389 and rs10758325, respectively); rs5065 and rs198389 were found to be in linkage disequilibrium. There was no association between genotype and age or sex for all three SNPs.

For all three SNPs, heterozygous genotype and homozygous minor allele genotype were combined in further analyses using regression models, because 1) relatively small proportions of homozygous minor allele genotype, and 2) heterozygous and homozygous minor allele genotype genotypes have been shown to behave similarly in terms of their effects in cardiovascular outcomes [7].

\section{Association between basic characteristics, obstructive CAD and average luminal narrowing}

Patients' age $(\mathrm{P}<0.01, \mathrm{RR}=1.20 / 10$ years $)$, male sex $(\mathrm{P}<$ $0.01, \mathrm{RR}=1.52)$, hypertension $(\mathrm{P}=0.03, \mathrm{RR}=1.23)$, type II

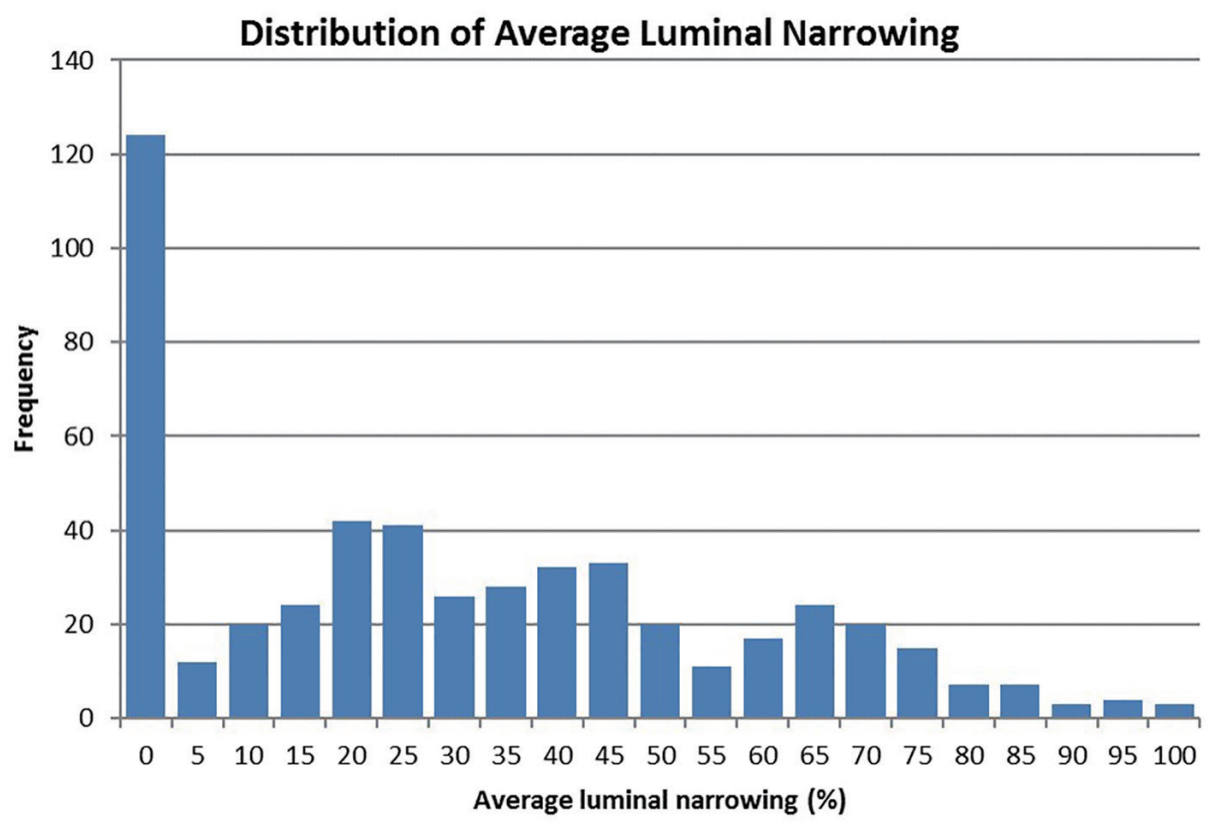

Figure 1. Distribution of average luminal diameter narrowing across four major coronary arteries $(n=513)$. 
Table 2. Natriuretic Peptide System SNP Genotype Frequencies

\begin{tabular}{|c|c|c|c|c|c|}
\hline \multirow{2}{*}{$\begin{array}{l}\text { SNP ID } \\
\text { (major allele/minor allele) }\end{array}$} & \multirow[b]{2}{*}{$\mathbf{n}$} & \multicolumn{4}{|c|}{ Genotypes } \\
\hline & & $\begin{array}{l}\text { Homozygous major allele } \\
(\mathrm{n}, \%)\end{array}$ & $\begin{array}{l}\text { Heterozygous } \\
(\mathrm{n}, \%)\end{array}$ & $\begin{array}{l}\text { Homozygous minor allele } \\
(\mathrm{n}, \%)\end{array}$ & MAF \\
\hline NPPB rs198389 (A/G) & 490 & $174(34.2 \%)$ & $257(50.5 \%)$ & $78(15.3 \%)$ & 0.42 \\
\hline NPR2 rs10758325 (G/A) & 507 & $176(34.7 \%)$ & $249(49.0 \%)$ & $83(16.3 \%)$ & 0.41 \\
\hline
\end{tabular}

MAF: minor allele frequency.

diabetes $(\mathrm{P}<0.01, \mathrm{RR}=1.31)$ and history of $\mathrm{MI}(\mathrm{P}<0.01, \mathrm{RR}$ $=1.39$ ) were associated with obstructive CAD. No association was found between obstructive CAD and smoking or family history of heart diseases.

Age $(\mathrm{P}<0.01, \beta=+5.39)$, the male sex $(\mathrm{P}<0.01, \beta=$ $+9.40)$, hypertension $(\mathrm{P}=0.02, \beta=+6.12)$ and type II diabetes $(\mathrm{P}<0.01, \beta=+8.78)$ were associated with increased average luminal narrowing. No association was found between average luminal narrowing and smoking, history of MI or family history of heart diseases.

\section{Association between natriuretic peptide SNPs and obstruc- tive CAD}

No association was observed between any natriuretic peptide SNPs and obstructive CAD (any one of four major coronary arteries with luminal narrowing of $70 \%$ or more) in the overall cohort. When sexes were divided, however, NPPB rs198389 homozygous major allele genotype (AA) was found to be associated with obstructive CAD in women only, with an RR of 1.50 (CI: $1.09-2.07, \mathrm{P}=0.014$ ) compared to AG or $\mathrm{GG}$ genotypes. The effect of rs198389 was also observed to be significantly different between the sexes $(\mathrm{P}=0.015)$, as no association was observed in the male cohort (Table 3 ).

\section{Association between natriuretic peptide SNPs and average luminal narrowing}

Among patients diagnosed with CAD (non-obstructive or obstructive; $\mathrm{n}=387$ ), NPPB rs198389 homozygous major allele genotype (AA) was found to be associated with increased average luminal narrowing $(\beta=4.91 \pm 2.25, \mathrm{P}=0.030)$ compared to AG or GG genotypes. When sexes were divided, this association was stronger in women $(\beta=11.05 \pm 3.92, \mathrm{P}=0.0051)$, but no association was observed in men (Table 4 ).

\section{Discussion}

In the current study, we did not find any association between NPPA rs5065 and NPR2 rs10758325 and CAD categories or overall coronary burden. However, we did find novel genderspecific association between $N P P B$ rs198389 and CAD. The rs198389 AA genotype was significantly associated with obstructive CAD and overall coronary burden in women but not men.

Minor allele of rs198389 affects NPPB promoter binding activity, resulting in higher circulating levels of BNP and NT-proBNP [9]. Despite of the research on NPPB rs198389 in hypertension, diabetes, left ventricular function and general cardiovascular outcome $[7,10]$, no previous study has directly tested the association between rs198389 and CAD. In the current study, we have investigated this association using coronary angiographic evidence, which is currently the clinical standard for determining CAD and quantifying the severity of atherosclerosis. In the overall cohort, none of rs198389 genotypes was associated with obstructive CAD category, but minor allele carriers (AG or GG) had significantly lower atherosclerotic burden than individuals with AA genotype based on average luminal narrowing. It suggests that while the AA genotype for rs198389 may aid in the development of atherosclerosis, its effect may not be enough to produce clinically significant single-

Table 3. Association of SNPs of the Natriuretic Peptide System and Obstructive CAD

\begin{tabular}{llllll}
\hline \multirow{2}{*}{ SNP } & Genotype & \multicolumn{4}{c}{ Obstructive CAD } \\
\cline { 3 - 6 } & & Overall RR (95\% CI) & Male RR (95\% CI) & Female RR (95\% CI) & P-value (sex difference) \\
\hline NPPA rs5065 $(\mathrm{n}=510)$ & AA & $1.04(0.88-1.22)$ & $0.99(0.83-1.16)$ & $1.31(0.88-1.95)$ & 0.199 \\
& AG/GG & Reference & Reference & Reference & 0.015 \\
NPPB rs198389 $(\mathrm{n}=509)$ & AA & $1.00(0.87-1.16)$ & $0.93(0.79-1.10)$ & $1.50(1.09-2.07)^{*}$ & 0.45 \\
& AG/GG & Reference & Reference & Reference & 0.443 \\
NPR2 rs10758325 $(\mathrm{n}=508)$ & GG & $1.02(0.88-1.18)$ & $1.04(0.90-1.22)$ & $0.90(0.63-1.28)$ & Reference \\
\hline
\end{tabular}

${ }^{*} \mathrm{P}<0.05$. Adjusted for age and sex. 
Table 4. Association of SNPs of the Natriuretic Peptide System and Average Luminal Narrowing (Excluding Patients With $0 \%$ Luminal Narrowing)

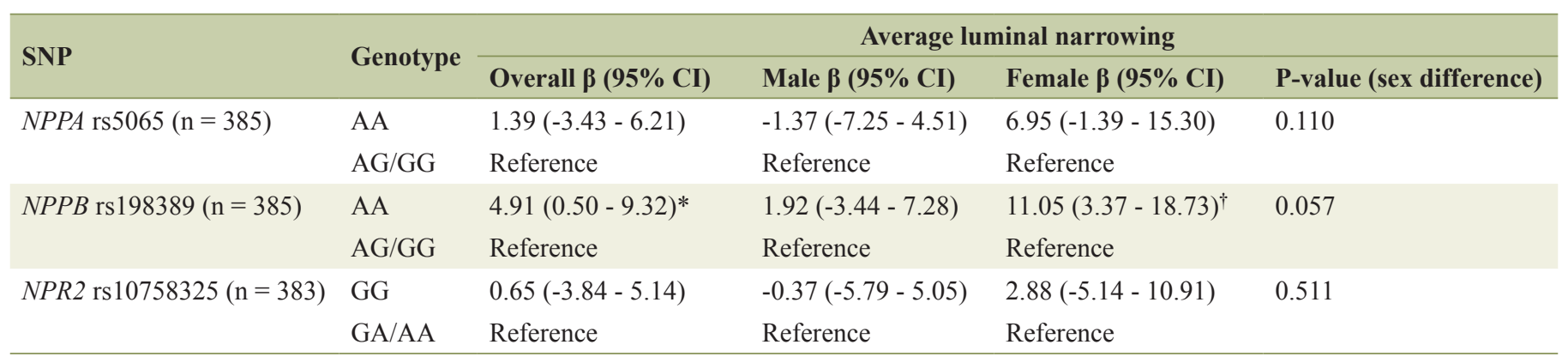

${ }^{*} \mathrm{P}<0.05 .{ }^{\mathrm{t} P}<0.01$. Adjusted for age and sex.

vessel disease, which the CAD categories are based on.

BNP's role in atherosclerosis pathophysiology is unknown. One of the possible pathways is through blood pressure regulation since hypertension is a prominent risk factor for CAD. However, current results do not support this as no association was found between rs198389 genotypes and hypertension.

More importantly, rs198389 genotypes had significantly different effects on CAD between sexes. Among women, the AA genotype was associated with higher risk of obstructive CAD and increased atherosclerotic burden, whereas no association was found in men. It suggests that the AA genotype for rs 198389 may be related to both overall atherosclerosis development and severe single-vessel disease. Furthermore, we may propose that BNP is significantly more involved in the pathophysiology of CAD in women than in men. It is possible that coronary atherosclerosis develops via different pathways in different sexes.

These sex-specific findings support the concept that CAD pathophysiology in women differs from that in men. Currently, diagnosis and prognosis of CAD in women are based on traditional risk scoring models, which were developed with studies where majority of subjects were men. This leads to a greater frequency of misclassification in women, who present CAD in sex-specific patterns. For example, non-obstructive CAD is more prevalent and has a poorer prognosis in women than in men $[3,4]$. The current findings suggest that integration of genetic markers may help to better stratify female CAD patients in the future.

Previously, numerous studies have attempted to establish an association between NPPA rs5065 and CAD, but have yielded opposing results. Some researchers found that the minor allele $\mathrm{G}$ was associated with higher risk of acute coronary syndrome [11] and greater severities of CAD [12], while other studies reported that the major allele A was more prevalent in CAD patients [13] and was associated with a higher risk of hypertension, more adverse ventricular remodeling and a higher rate of hospital readmission [7]. The discrepancy could be explained by diverse patient populations and different outcomes defining CAD. With regard to whether major allele or minor allele of NPPA rs5065 is deleterious in CAD, the current study could not support these findings either way, as we did not find any association between NPPA rs5065 genotypes and CAD categories or atherosclerotic burden.
The current study is limited by its relatively small sample size, especially of the female cohort. Future endeavors should focus on recruiting a much larger female cohort or a femaleonly population. Other angiographic scoring system, such as the Gensini score, could be incorporated in future studies to confirm current results and validate the method of average luminal narrowing. Lastly, natriuretic peptide SNPs, especially $N P P B$ rs 198389, could be added into traditional risk stratification and diagnostic tools in order to test whether they would improve predicative values.

\section{Conclusion}

In summary, the current study investigated the association between three natriuretic peptide SNPs and CAD through angiographic evidence in a Canadian population. We identified $N P P B$ rs 198389 minor allele associated with a lower coronary atherosclerotic burden. This polymorphism was also shown to be associated with a reduced risk of obstructive CAD only in women, suggesting that BNP has different involvements in the pathophysiology of CAD between sexes. These findings provide further evidence for an important protective role of BNP in CAD development, and support the notion that CVDs in women differ from those in men at the molecular level.

\section{Financial Disclosures}

None.

\section{Grant Support}

This study was supported by the Heart and Stroke Foundation of Canada (HSFC) the Canada Foundation for Innovation (CFI) and the Garfield Kelly Cardiovascular Development Fund, Queen's University.

\section{References}

1. Shaw LJ, Shaw RE, Merz CN, Brindis RG, Klein LW, 
Nallamothu B, Douglas PS, et al. Impact of ethnicity and gender differences on angiographic coronary artery disease prevalence and in-hospital mortality in the American College of Cardiology-National Cardiovascular Data Registry. Circulation. 2008;117(14):1787-1801.

2. Johnson BD, Shaw LJ, Buchthal SD, Bairey Merz CN, Kim HW, Scott KN, Doyle M, et al. Prognosis in women with myocardial ischemia in the absence of obstructive coronary disease: results from the National Institutes of Health-National Heart, Lung, and Blood Institute-Sponsored Women's Ischemia Syndrome Evaluation (WISE). Circulation. 2004;109(24):2993-2999.

3. Izadnegahdar M, Mackay M, Lee MK, Sedlak TL, Gao M, Bairey Merz CN, Humphries KH. Sex and Ethnic Differences in Outcomes of Acute Coronary Syndrome and Stable Angina Patients With Obstructive Coronary Artery Disease. Circ Cardiovasc Qual Outcomes. 2016;9(2 Suppl 1):S26-35.

4. Pepine CJ, Ferdinand KC, Shaw LJ, Light-McGroary KA, Shah RU, Gulati M, Duvernoy C, et al. Emergence of Nonobstructive Coronary Artery Disease: A Woman's Problem and Need for Change in Definition on Angiography. J Am Coll Cardiol. 2015;66(17):1918-1933.

5. Volpe M, Carnovali M, Mastromarino V. The natriuretic peptides system in the pathophysiology of heart failure: from molecular basis to treatment. Clin Sci (Lond). 2016;130(2):57-77.

6. Yancy CW, Jessup M, Bozkurt B, Butler J, Casey DE, Jr., Drazner MH, Fonarow GC, et al. 2013 ACCF/AHA guideline for the management of heart failure: a report of the American College of Cardiology Foundation/American Heart Association Task Force on Practice Guidelines. J Am Coll Cardiol. 2013;62(16):e147-239.
7. Ellis KL, Newton-Cheh C, Wang TJ, Frampton CM, Doughty RN, Whalley GA, Ellis CJ, et al. Association of genetic variation in the natriuretic peptide system with cardiovascular outcomes. J Mol Cell Cardiol. 2011;50(4):695-701.

8. Dupont WD, Plummer WD, Jr. Power and sample size calculations. A review and computer program. Control Clin Trials. 1990;11(2):116-128.

9. Johansson A, Eriksson N, Lindholm D, Varenhorst C, James S, Syvanen AC, Axelsson T, et al. Genome-wide association and Mendelian randomization study of NTproBNP in patients with acute coronary syndrome. Hum Mol Genet. 2016;25(7):1447-1456.

10. Meirhaeghe A, Sandhu MS, McCarthy MI, de Groote P, Cottel D, Arveiler D, Ferrieres J, et al. Association between the T-381C polymorphism of the brain natriuretic peptide gene and risk of type 2 diabetes in human populations. Hum Mol Genet. 2007;16(11):1343-1350.

11. Barbato E, Bartunek J, Mangiacapra F, Sciarretta S, Stanzione R, Delrue L, Cotugno M, et al. Influence of rs5065 atrial natriuretic peptide gene variant on coronary artery disease. J Am Coll Cardiol. 2012;59(20):1763-1770.

12. Ziaee S, Kalayinia S, Boroumand MA, Pourgholi L, Cheraghi S, Anvari MS, Sheikhvatan M. Association between the atrial natriuretic peptide rs5065 gene polymorphism and the presence and severity of coronary artery disease in an Iranian population. Coron Artery Dis. 2014:1.

13. Larifla L, Maimaitiming S, Velayoudom-Cephise FL, Ferdinand S, Blanchet-Deverly A, Benabdallah S, Donnet JP, et al. Association of 2238T $>$ C polymorphism of the atrial natriuretic peptide gene with coronary artery disease in Afro-Caribbeans with type 2 diabetes. Am J Hypertens. 2012;25(5):524-527. 